\title{
Epidemiology of Brucellosis in Small Ruminants
}

\section{Bidjeh Kebkiba ${ }^{1 *}$, Adoum Gaye ${ }^{1}$, Adam Hassan Yacoub ${ }^{2}$ and Bouzabo Patchilii ${ }^{3}$ \\ Taye, Adam Hassan Yacoub2 and Bouzabo}

${ }^{1}$ Livestock Research Institute for Development (LRID), Republic of Chad

${ }^{2}$ General Inspection, Ministry of Livestock and Animal Productions, Republic of Chad

${ }^{3}$ Regional Center of Animal Health for central Africa, Republic of Chad

*Corresponding author: Bidjeh Kebkiba, Livestock Research Institute for Development (LRID),

Farcha street, P.O. Box 433, N’Djamena, Republic of Chad, Tel: +23563759045; Email: bidjehkebkiba@yahoo.fr

\section{Abstract}

Brucellosis is a zoonotic infectious disease caused by a bacterium of the genus Brucella, common to certain animals, both domestic and wild, and to humans. Historically, this disease is known as Malta fever or melitococcal disease. The causative agent was isolated in 1887 on the Island of Malta by David Bruce. Its extension is worldwide with a predominance in developing countries. The determinants of the epidemiology of the disease in small ruminants in sub-Saharan Africa are not well understood and the available data are fragmentary and sometimes insufficient. This bacterial infection affects thousands of people around the world. The main clinical signs of brucellosis in small ruminants are abortion, retained placenta, stillbirth, orchitis and arthritis. Infected animals, milk and dairy products are the sources of infection in humans. B. melitensis, B. abortus and $B$. suis are the most important species in terms of public health and economy. Pigs are the source of almost all human contaminations. B. melitensis, the species most frequently implicated in human pathology, is largely predominant in sheep and goats. This organism contains three biovars and all of them can cause disease in small ruminants. The purpose of this article is to summarize the epidemiological data collected from bibliographic references: the causative agent of the disease, clinical manifestations, sources and modes of transmission of infection, diagnosis and prophylaxis.

Keywords: Epidemiology; Brucellosis ; Small Ruminants; Goats, Sheep; Zoonosis

\section{Introduction}

Small ruminants (goats and sheep) play an important role in livestock systems around the world. They are prolific and hardy animals that adapt more easily to the difficult conditions encountered in the Sahelian zone. The breeding of these animals constitutes a significant nutritional contribution because their milk and meat provide an important part of the protein ration of rural populations. In Africa, they constitute cash that can be easily mobilized for current expenditure. Finally, they allow breeders in developing countries a capitalization often used during an epizootic phenomenon decimating the cattle herd such as the episode of rinderpest (1983-1984). In this context, the production problems of ewes and goats constitute a factor limiting the profitability of this breeding [1].

Even though the livestock sub-sector constitutes an important part of the national economy in some African countries, its development is hampered by various constraints such as infectious diseases like brucellosis and many others. Indeed, brucellosis is one of the main constraints and the major concern of the production of small ruminants in the world in general and in Africa in particular. There are many infectious 


\section{Open Access Journal of Microbiology \& Biotechnology}

diseases of animals and many of them are of public health significance, resulting in morbidity and mortality [2]. These diseases are the main causes of economic losses in animal production. Among these, we can mention brucellosis, which is a highly infectious disease of humans and many animal species. It is important from a public health perspective as well as from an economic perspective [3]. Brucellosis remains one of the most common zoonotic diseases with more than 50,000 cases reported annually worldwide [4]. Although the disease has been eradicated in most developed countries, its incidence is increasing in developing countries [5]. The disease affects pets, wildlife and humans and is caused by organisms called Brucella. It affects the reproductive system of animals, leading to losses in productivity, such as reduced milk production, abortion, weak offspring, weight loss, early culling and condemnation of infected animals due to infertility and lameness [6]. It constitutes a hindrance to internal trade and exports. The objective of this manuscript is to summarize from the bibliographical references some epidemiological data on brucellosis in general and that of small ruminants in particular.

\section{Epidemiology}

\section{Causative Agent of the Disease}

Brucellosis had become a problem for the British garrison in Malta with significant morbidity and mortality among British soldiers. Dr. David Bruce, a medical officer has been dispatched to the Island for this purpose to clarify the situation. He had coordinated a team of scientists who succeeded in isolating Micrococcus Melitensis in 1887 as a causative agent of the disease from raw goat's milk consumed by military personnel. This bacterium will later bear its name, Brucella melitensis. Other Brucella species include $B$. abortus isolated by Bang in 1897 and B. suis described by Traum in 1914. These 3 species are the most important in terms of public health and economy. There are several other species, including B. ovis, B. canis, B. neotomae, B. microti, $B$. Epidimytis and at least 2 species: B. ceti and B. pinnipedialis, which infect marine mammals but are also potentially pathogens for humans.

According to Refai $\mathrm{M}$, et al. [7], three closely related species of the genus brucella are generally known as the causative agent of brucellosis: B. abortus, B. melitensis and $B$. suis. Based on genetic and epidemiological elements, twelve (12) species of Brucella have been identified. $B$. melitensis, B. abortus and B. suis species are subdivided into biovars. Indeed, brucellosis is an anthropozoonosis (disease transmitted to humans from animals); this disease is caused by coccobacilli (bacteria) of the genus Brucella, named after David Bruce. These bacteria of the genus Brucella mainly infect cattle, sheep, goats, pigs and humans.

\section{Susceptible Animals}

Globally, Brucella mainly infect ruminants (cattle, goats and sheep); dromedaries and pigs are responsible for almost all human contamination. This animal reservoir has spread to aquatic mammals such as dolphins, seals and certain river fish [8]. Note that the preferential adaptation of a bacterial species to one or more animal species is only relative [9]. However, B. melitensis, the species most frequently implicated in human pathology, is largely predominant in sheep and goats. This organism is a Gram-negative facultative intracellular pathogen and contains three biovars $(1,2$ and 3 ). All of these biovars can cause disease in small ruminants [10]. Brucella Abortus naturally infects cattle, but can also infect other domestic ruminants (zebus, bison, sheep and goats, reindeer), suidae, equines and carnivores, etc.) and wild animals (buffaloes, deer, etc.), rodents $[8,11]$. Note that bovine brucellosis can also be caused by $B$. melitensis or $B$. suis. Despite the apparent host, there is some specificity of $B$. melitensis to goats. This bacterium also infects dromedaries [12-15]. Dromedaries (Camelus dromedarius) are found in the deserts of sub-Saharan and northern Africa, from the Middle East to northern India, an area where they are essential for meat, milk, leather, wool and transport [16]. Dromedary brucellosis was first reported in 1931 and has since been recorded in all dromedary herding countries in this region, but is particularly well documented in infected herds in Africa and the Arabian Peninsula [12]. Dromedaries are not a primary host for Brucella spp, but infections with $B$. melitensis occur due to the mixing of these animals and small ruminants in a herd [14]. In fact, determining the genetic diversity of $B$. melitensis in dromedaries would provide valuable information on the spread and transmission of the disease in goats and sheep in endemic areas, and particularly would help to better understand the role of camels in human infections. Traditionally found in goats and sheep, Brucella melitensis is increasingly recognized as infecting camels [13]. To date, most studies of brucellosis in camels have focused on serological surveys, providing only a limited understanding of the molecular epidemiology of circulating strains.

The association of B. abortus with abortion in sheep has been demonstrated in several countries by the isolation of microorganisms [17-20]. The spectrum of pathogenicity of Brucella, at least for the major species, is extremely broad: $B$. Abortus, B. Melitensis and B. suis can naturally infect humans, domestic and wild ruminants, suidae, equines, carnivores, rodents and sometimes birds. This lack of host specificity explains the interdependence that may exist between brucellosis in various animal species and the epidemiological consequences that result from it. 


\section{Open Access Journal of Microbiology \& Biotechnology}

\section{Clinical Manifestations}

Brucellosis manifests itself in several forms in animals and humans: the main clinical signs of brucellosis are abortion, retained placenta, stillbirth, orchitis, arthritis in animals, and undulating fever in humans male [7]. In cattle, the most common form is abortion (2-4 months after infection), knee hygroma; weak calves; decreased lactation, retention of the placenta, temporary or permanent infertility, orchitis in bulls.

In small ruminants, the clinical signs of brucellosis are manifested mainly by abortions [21]. In ewes, abortion occurs in the $3^{\text {rd }}$ month of gestation; placental retention is less common than in cattle. Sometimes you notice temporary sterility even in the absence of retained placenta. It can affect $10 \%$ of ewes in a flock in the first year of infection. Mastitis is often seen in sheep. Unlike cattle, brucellic mastitis can affect many herds. In rams, the infection is usually invisible; however, it is possible to observe cases of orchitis, epididymitis or reduced fertility. In humans, symptoms are nonspecific and are easily confused with other fever-causing illnesses such as malaria, typhoid fever, rheumatic fever, and osteoarthritis. In addition, the working capacity is reduced due to the disease caused by Brucella.

\section{Sources of Infection and Modes of Transmission}

Any animal that is infected, sick or apparently healthy is a potential source of brucella. It can also remain a carrier of the germ and contagious throughout its period of exploitation; Therefore, any infected animal should be considered a possible source of brucella. Consequently, any control action must take into account not only the presence of the sick but also that of the carriers of the germs. Trade, loans from rams and especially transhumance play an important role in the contamination of free herds and the transmission of the disease in a farm or even in a country. The stays of animals in contaminated pastures or sheepfolds are also to blame in the contamination of animals by brucella.

Brucellosis usually spreads around the time of abortion or childbirth. High concentrations of bacteria are found in fetal fluids from an infected animal. Sources of infection for animals include runts, vaginal discharge, milk and semen from infected animals. Transmission in wildlife occurs through spillage from domestic animals and wildlife [22]. Contact between wildlife, livestock and humans is common in pastoral and agro-pastoral communities in sub-Saharan Africa. This interaction promotes disease transmission between these animals (wildlife and livestock) and humans. Infected domestic and wild animals and their products are the source of infection for humans. The disease is an occupational risk for farmers, practicing veterinarians and workers in the meat industry [23]. Transmission usually occurs through ingestion of contaminated food and water, vaginal exudates, sores on the skin, conjunctiva, mammary gland and coitus. Bacteria can be transmitted to humans by butchering and consuming raw milk and undercooked products. A recent report from Botswana mentions that household bushmeat processing practices pose a risk of exposure to Brucella spp for family members and the entire community [24]. As with brucellosis in other animals, these abortion events spread the bacteria widely and allow transmission to other livestock and to livestock owners [24].

\section{Geographical Distribution}

Practically brucellosis is prevalent all over the world. It is one of the most common zoonoses in the world and is endemic in most African countries. But their geographic distribution varies considerably [3]. Its prevalence is highest where domestic animals and humans coexist [7]. This disease is widespread in Africa where it remains one of the most important zoonotic diseases. It is mainly confined to countries where small ruminants are raised extensively. It is a common disease of cattle in the Middle East and in sub-Saharan and northern Africa, but remains very poorly described in the region both genetically and epidemiologically. This disease is a public health problem throughout the Middle East [25]. The presence of an African lineage in North Africa and the Arabian Peninsula indicates the interdependence of cattle in the Greater Middle East, possibly due to movements of animals through trade and transhumance between the two regions, although that the Mediterranean lineage still predominates in the sub-region.

\section{Diagnosis}

Clinical Diagnosis: The clinical diagnosis is still insufficient. Only laboratory tests can provide a definite diagnosis. From a general point of view, brucellosis should be suspected in the presence of damage to the reproductive organs resulting in abortions (serial or sometimes sporadic) and in males by orchitis and epididymitis [26]. These symptoms can coexist with joint involvement (arthritis) or bursa (bursitis).

Laboratory Diagnostic: The "Manual of Diagnostic Tests and Vaccines for Terrestrial Animals" published by the OIE lists diagnostic tests in two categories: prescribed tests and alternative tests [27]. The prescribed tests are required by the OIE Terrestrial Animal Health Code for the International Movement of Animals and Animal Products and are considered optimal for determining the health status of animals. As recommended by the OIE, these tests should be validated and antigens should be purchased from Credible Sources. A control certificate of the purchased lot issued by a competent control authority should always be provided. The use of tests, which do not meet these requirements 


\section{Open Access Journal of Microbiology \& Biotechnology}

may generate unreliable results and thus may lead to errors in epidemiological interpretation. The OIE emphasizes the use of prescribed tests for trade to avoid transmission of the disease across borders [27]. Laboratory diagnosis of brucellosis includes : microbiological examination, serological testing (agglutination reaction, complement fixation, immunofluorescence, etc.) and bioassay (inoculation into laboratory animals). Nowadays, a definitive diagnostic technique is not yet available, although it has been pursued for over a century.

The microorganism can be recovered from a variety of materials. The placenta being the most infectious and with the greatest concentration of bacteria, followed by lymph nodes and milk in goats and sheep, blood in humans [28]. Most of Brucella strains are slow growing, some of them require serum enriched culture media, and even experienced labs only report isolation rates between $20-50 \%$ [29]. The diagnosis of brucellosis is usually made by a combination of methods. In addition, the presumptive diagnosis can be made by the use of several serological tests specific to Brucella antibodies, but an unequivocal diagnosis requires the bacteriological demonstration of the microorganism hence the collection and shipment of suitable samples to the laboratory are a great importance.

Sample: Brucella can be isolated from the samples of choice. These materials include the contents of the stomach, spleen and lungs, aborted fetuses, fetal placentas and membranes, vaginal swabs, milk, semen, joint fluids or hygroma from adult animals. Of animal carcasses, the preferred tissues for culture are mammary, lymph nodes (medial and internal iliac, retropharyngeal, parotid and prescapular) and spleen [7].

Prophylaxis: Prevention of the disease is essential given its risk to human health and its significant economic impact. According to Refai M, et al. [7], the eradication of ovine and caprine brucellosis is feasible through massive annual vaccination of these animals coupled with sanitary measures. The advantage of implementing mass vaccination campaigns as well as the negative consequences of unsuccessfully implementing or stopping such vaccination campaigns are illustrated by the work carried out in Mongolia and Greece [27]. The sanitary measures for the control of brucellosis indicated in the public health policy vary from country to other; For example, in developed countries, these measures consist of systematically slaughtering animals that have reacted positively to serological tests. The isolation of parturients, the disinfection of premises and equipment, the destruction of potential virulent materials (abortions, placentas, etc.) complete the action of screening and slaughtering positive animals.
Medical prophylaxis relies on the use of vaccines. This act is justified in highly infected regions because it represents the only economically usable method. On the other hand, it should be avoided in an uninfected or slightly infected region [27,30-34]. However, taking into account the fact that brucellic immunity is relative, Vaccination is not a sufficient guarantee : it can only be envisaged in animals if it does not induce post-vaccine antibodies likely to be interfere with serological screening for infection. Around the world, there are inactivated and attenuated vaccines. But whatever the type of vaccine, it must meet certain requirements which are: safety, efficacy and absence of antibody production. Among the many vaccines that can be used, we can mention those that are commonly used in the world:

- REV1 vaccine (attenuated vaccine)- It is prepared from a streptomycin-dependent mutant of B. melitensis biovar1 in phase $\mathrm{S}$. This vaccine is the most effective and widely used in the world in small ruminants.

- H38 vaccine (inactivated vaccine)- It is prepared from strain H38 (strain of $B$. melitensis, biovar1 in phase $\mathrm{S}$ inactivated by formalin and adjuvanted with an oily excipient. This vaccine has a strong immunogenicity.

\section{Conclusion}

Brucellosis is an infectious disease that affects both domestic and wild animals. It can be transmitted to humans through the consumption of infected products (e.g. raw milk). The most common species in Africa as well as in the Arabian Peninsula is Brucella melitensis. It is this species that affects sheep, goats and camels. It is also transmitted to humans. The target and vulnerable populations are: breeders, slaughterhouse staff, veterinarians and diagnostic laboratory staff. The application of sanitary measures combined with mass vaccination campaigns makes it possible to control or eradicate animal and human brucellosis in a country.

\section{References}

1. Carpenter C, Hubert WT (1963) Brucellosis: In diseases transmittted from animals to man. $5^{\text {th }}$ (Edn.), Illinois, USA.

2. Pal M (2005) Importance of zoonoses in public health. Indian Journal of Animal Sciences 75: 586-591.

3. Pal M (2007) Zoonoses. $2^{\text {nd }}$ (Edn.), Satyam Publishers, Jaipur, India.

4. Hadush A, Pal M (2013) Brucellosis-an infectious reemerging bacterial zoonosis of global significance. Int J Livest Res 3(1): 28-34.

5. Seifert HSH (1996) Diseases caused by aerobic rods. 1 Brucellosis. In: Bokma BH, et al. (Eds.), Tropical animal 


\section{Open Access Journal of Microbiology \& Biotechnology}

health, Kluwer Academic, Dordrecht, pp: 356-367.

6. Trilenko PA (1976) La brucellose animale Leningrad, Kolos.

7. Refai M (2002) Incidence and control of brucellosis in the Near East region. Vet Microbiol 90(1-4): 81-110.

8. Maurin M (2005) Brucellosis at the dawn of the $21^{\text {st }}$ century. Med Mal Infect 35(1): 6-16.

9. Vanderkerckhove C, Stahl JP (1993) Brucellosis. Epidemiological data and theuraptic Rev Prat 7: 47-52.

10. Chakroun M, Bouzouaia N (2007) La brucellose: a still relevant zoonosis. Rev Tun Infectiol 1(2): 1- 10.

11. Fyumagwa RD, Wambura PN, Mellau LSB, Hoare R (2009) Seroprevalence of Brucella abortus in buffaloes and wildebeests in the Serengeti ecosystem: A threat to humans and domestic ruminants. Tanzan Vet J 26(2): 6267.

12. Gwida M, El Gohary A, Melzer F, Khan I, Rosler U, et al. (2012) Brucellosis in camels. Res Vet Sci 92(3): 351-355.

13. Musa MT, Eisa MZ, El Sanousi EM, Wahab AMB, Perrett L (2008) Brucellosis in camels (Camelus dromedarius) in Darfur, Western Sudan. J Comp Pathol 138(2-3): 151155.

14. Sprague LD, Al Dahouk S, Neubauer H (2012) A review on camel brucellosis: a zoonosis sustained by ignorance and indifference. Pathog Glob Health 106(3): 144-149.

15. Wernery U (2014) Camelid brucellosis : a review. Rev Sci Tech 33(3): 839-857.

16. Wilson RT (1984) The Camel. Longman Group Limited, Harlow, Essex, UK, pp: 223.

17. Allsup TN (1974) Failure to demonstrate Brucella infection in ewes exposed to natural bovine infection. Vet Rec 94(9): 183-186.

18. Bannatyne CC (1960) Brucella abortus infection in black face ewe. Vet Rec 72: 660-661.

19. Luchsinger DW, Anderson RK (1967) Epizootiology of brucellosis in a flock of sheep. J Am Vet Med Assoc 150(9): 1017-1021.

20. Merieux R (1991) Labrucellose, France.

21. Okoh AE (1980) Abortion in sheep near Kano, Nigeria. Trop Anim Health Prod 12(1): 11-14.

22. Mangen MJ, Otte J, Pfeiffer D, Chilonda P (2002) Bovine brucellosis in Sub-Saharan Africa : Estimation of seroprevalence and impact on meat and milk offtake potential. In: Livestock Policy Discussion Paper No. 8.
Food and Agriculture Organization, Livestock (FAO) Information and Policy Branch, AGAL, pp: 1-53.

23. WHO (2015) Brucellosis in humans and animals. World Health organization, Geneva 27, Switzerland.

24. Acha A, Szyfres B (2003) Zoonoses and Communicable Diseases Common to Man and Animals. $3^{\text {rd }}$ (Edn.), Pan American Health Organization, Washington, DC, USA.

25. Pappas G, Memish ZA (2007) Brucellosis in the Middle East: a persistent medical, socioeconomic and political issue. J Chemother 19(3): 243-248.

26. OIE AHS (2012) Manual of Diagnostic Tests and Vaccines for Terrestrial Animals, Chapter 2.4.3 $7^{\text {th }}$ (Edn.), Bovine brucellosis. Paris: Office International des Epizooties, paris, France.

27. Zowghi E, Ebadi A, Mohseni B (1990) Isolation of brucella organisms from the milk of seronegative cows. Rev Sci Tech 9(4): 1175-1178.

28. Gwida MM, El Gohary AH, Melzer F, Tomaso H, Rosler $\mathrm{U}$, et al. (2011) Comparison of diagnostic tests for the detection of Brucella spp. In camel sera. BMC Res Notes 4: 525 .

29. Lancelot R, Imadine M, Mopate Y, Faye B (1993) The Ecopathological Investigation on Goat Pneumopathies In the cold dry season In Chad: Methodological Aspects. Rev Elev Med Vet Tropical countries 46(3): 485-494.

30. Godfroid J (2002) Brucellosis in wildlife. Rev Sci Tech 21(2): 277-286.

31. Bouzouaia N, Chakroun M, Rachdi J, Rachdi T (1995) Epidemiological, clinical and therapeutic aspects of brucellosis in Tunisia Apropos of epidemics in Gafsa. Tunis Med 73(11): 443-448.

32. Memish ZA, Mah MW (2001) Brucellosis in laboratory workers at a Saudi Arabian hospital. Am J Infect Control 29(1): 48-52.

33. Mumah GJ, Marcottyi T, Pfeiffer D, Skjervek E, Pappas G, et al. (2013) A One Health surveillance and control of brucellosis in developing countries : Moving away from improvisation. Comp Immunol Microbiol Infect Dis 36(3): 241-248.

34. Allsup TN (1969) Abortion in sheep associated with Brucella abortus infection. Vet Rec 84(5): 104-108. 